\title{
Uso de EVA Modificado com Grupos Mercaptana na Compatibilização de Misturas NBR/EPDM
}

\author{
Marcia G. Oliveira, Bluma G. Soares \\ Instituto de Macromoléculas Professoroa Eloisa Mano, UFRJ
}

Resumo: Copolímeros de etileno - acetato de vinila com diferentes teores de acetato de vinila - foram funcionalizados com grupos mercaptana e utilizados como agentes compatibilizantes reativos em misturas envolvendo borracha nitrílica e copolímeros de etileno-propileno-dieno (EPDM). Foi investigada a influência das características estruturais dos copolímeros funcionalizados no processo de vulcanização, propriedades mecânicas e dinâmico-mecânicas bem como resistência ao envelhecimento. A adição de pequena porção desses copolímeros resultou em tempos de cura menores. Copolímeros funcionalizados, obtidos a partir da transesterificação de EVA com ácido mercaptoacético (EVASH), apresentaram desempenho melhor como compatibilizante do que aquele obtido a partir da esterificação de EVA hidrolisado com o mesmo ácido (EVALSH). As misturas compatibilizadas com o primeiro sistema apresentaram melhores propriedades mecânicas além de uma morfologia mais refinada e uniforme. A presença dos grupos mercaptana nesses agentes compatibilizantes resultou em uma melhora na resistência ao envelhecimento das misturas.

Palavras-chave: Misturas elastoméricas, compatibilização reativa, borracha nitrílica, EPDM, copolímeros de EVA.

\section{The Use of Mercapto-Modified EVA as Compatibilizing Agent for NBR/EPDM Blends.}

Abstract: Ethylene-vinyl acetate (EVA) copolymers with different amounts of vinyl acetate have been functionalized with mercapto groups and these reactive compounds were used as compatibilizing agent for nitrile rubber (NBR)/ ethylene-propylene-diene rubber (EPDM) blends. The effects from the structural properties of mercapto-modified EVA compounds on the vulcanizing characteristics, mechanical properties, aging resistance and dynamic-mechanical properties were investigated. The addition of low amounts of mercapto-modified EVA resulted in a decrease of curing time. The functionalized copolymer obtained through transesterification reaction between mercaptoacetic acid and EVA (EVASH) presented better compatibilizing action than that obtained from esterification of hydrolyzed EVA and mercaptoacetic acid (EVALSH). Blends compatibilized with the former compound presented better mechanical performance and more refined morphologies. Finally, aging resistance was improved by the use of mercapto-modified EVA.

Keywords: Elastomer blends, reactive compatibilization, nitrile rubber, EPDM, EVA copolymer .

\section{Introdução}

Misturas envolvendo borracha nitrílica (NBR) e terpolímero de etileno-propileno-dieno (EPDM) têm sido estudadas, com o objetivo de desenvolver materiais que associem as boas propriedades e a excelente resistência a óleos da NBR, a uma maior resistência ao envelhecimento.

NBR e EPDM apresentam incompatibilidade termodinâmica e diferem consideravelmente quanto à polaridade, o grau de insaturação e a velocidade de cura. A vulcanização deste sistema ocorre de maneira heterogênea, principalmente quando o enxofre é utilizado como agente de cura. Dois fatores contribuem

Autor para correspondência: Bluma G. Soares, Instituto de Macromoléculas Professora Eloisa Mano, UFRJ, Caixa Postal 68525, CEP: 21945-970, Rio de Janeiro, RJ. E-mail: bluma@ima.ufrj.br 
para este comportamento: (i) a diferença de reatividade dos elastômeros; e (ii) a diferença de solubilidade dos agentes de cura nos elastômeros. Ambos fatores atuam de forma a favorecer a migração dos agentes de cura do EPDM para a NBR. Este tipo de incompatibilidade não é facilmente superado e muitas vezes exige o desenvolvimento de um sistema de vulcanização específico $^{[1-3]}$. Na literatura ${ }^{[3]}$ encontram-se basicamente dois tipos de estudo sobre esta mistura, os que envolvem investigações sobre o sistema de vulcanização e os que envolvem o uso de agentes compatibilizantes.

Dentre as alternativas estudadas para solucionar o problema da vulcanização heterogênea destacam-se: modificações na cadeia hidrocarbônica dos aceleradores $^{[3]}$, substituição do óxido de zinco por óxido de chumbo $^{[4]}$, graftização de aceleradores na cadeia do elastômero menos insaturado ${ }^{[5]}$, vulcanização com peróxidos e resinas $^{[6]}$.

Alguns agentes interfaciais já foram empregados na compatibilização da mistura NBR/EPDM, dentre os quais destacam-se o poli(trans-octeno) $(\mathrm{TOR})^{[7]}$, o polietileno clorado (CPE) e o polietileno clorosulfonado ${ }^{[8-12]}$. Todos estes produziram misturas com morfologia refinada e melhores propriedades mecânicas.

A compatibilização de misturas de elastômeros com diferentes teores de insaturação tem sido também conduzida na presença de copolímeros funcionalizados com grupos mercaptana. Tais grupos funcionais são capazes de reagir com as ligações duplas da fase elastomérica mais insaturada, promovendo assim uma boa adesão entre as fases da mistura. Resultados anteriores envolvendo a adição de EVA funcionalizado com grupos mercaptana em misturas NR/EVA ${ }^{[13-17]}$, NBR/EVA ${ }^{[18]}$ e SBR/EVA ${ }^{[19]}$ revelaram uma melhora na adesão interfacial e consequentemente no comportamento mecânico destas misturas. A partir desses resultados, resolveu-se então investigar a influência de EVA funcionalizado com grupos mercaptana na compatibilização do sistema NBR/EPDM. Para esses estudo, foram sintetizadas diferentes amostras de EVA funcionalizado, utilizando-se diferentes rotas sintéticas e amostras de EVA com diferentes teores de acetato de vinila.

\section{Experimental}

Materiais

NBR N-615 B, foi cedida pela Nitriflex, contendo
$33 \%$ em peso de acrilonitrila e com viscosidade Mooney $\mathrm{ML}_{1+4}=48$. EPDM foi cedido pela DSM, contendo $60 \%$ em peso de etileno e $1,141 \mathrm{mmol} / \mathrm{g}$ de etilideno-norborneno (ENB) e com viscosidade Mooney $\mathrm{ML}_{1+4}=90$. EVA contendo $18 \%$ em peso de acetato de vinila (EVA18) foi cedido pela Petroquímica Triunfo S. A. As amostras de EVA contendo 28 (EVA28) e 33\% em peso (EVA33) de acetato de vinila foram cedidas pela Politeno Ind. Com. Ácido mercaptoacético foi adquirido pela Vetec Ind. Químicas S.A. Óxido de zinco, ácido esteárico, enxofre, 2,2-ditiomercaptobenzotiazol (MBTS) e dissulfeto de tetrametiltiuram (TMTD), todos de grau comercial.

\section{Funcionalização do EVA}

Rota 1: A primeira rota consistiu da hidrólise total do EVA18 e posterior esterificação do produto com ácido tioglicólico, segundo processo descrito na literatura $^{[20]}$. A hidrólise total do EVA foi realizada em solução metanólica a $10 \%$ em peso de $\mathrm{NaOH}$ a $70{ }^{\circ} \mathrm{C}$ durante $2 \mathrm{~h}$, dando origem ao poli(etileno-coálcool vinílico) (EVAL). A hidrólise total foi determinada a partir de análise termogravimétrica, segundo a literatura ${ }^{[20]}$. EVA hidrolisado foi, então, esterificado com ácido tioglicólico em tolueno a $110{ }^{\circ} \mathrm{C}$ durante $2 \mathrm{~h}$, dando origem ao EVALSH. A Figura 1 ilustra o esquema total da reação.

Rota 2: Amostras de EVA contendo 18, 28 e $33 \%$ de acetato de vinila foram dissolvidas em tolueno na concentração de $10 \%$ em peso e tratadas com ácido tioglicólico a $110^{\circ} \mathrm{C}$ durante $6 \mathrm{~h}$, dando origem ao EVASH18, EVASH28 e EVASH33, respectivamente, conforme ilustra a Figura 2.

Os agentes compatibilizantes foram caracterizados por análise de enxofre e possuíam praticamente a mesma quantidade de enxofre, cerca de $6 \%$ em peso.

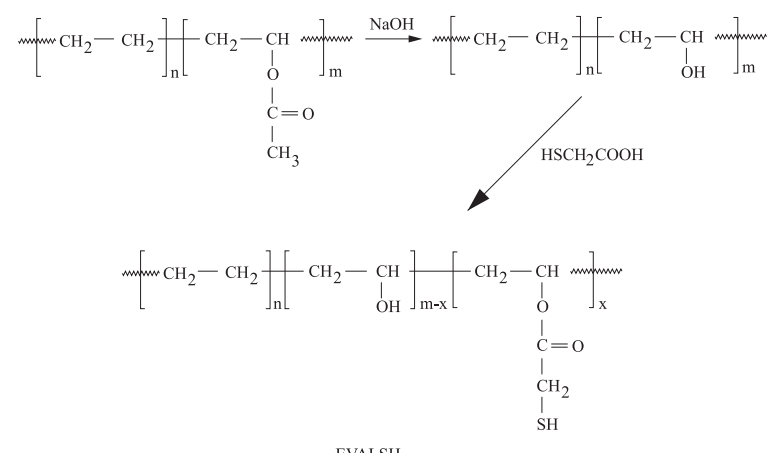

Figura 1. Esquema de funcionalização do EVA para obtenção do EVALSH (rota 1) 
Figura 2. Esquema de funcionalização do EVA para obtenção do EVASH (rota 2)

Tabela 1: Formulação da mistura NBR/EPDM

\begin{tabular}{lc}
\hline & phr \\
\hline NBR & 70 \\
EPDM & 30 \\
Agente compatibilizante & 5,0 \\
Óxido de zinco & 5,0 \\
Ácido esteárico & 0,5 \\
Enxofre & 1,0 \\
MBTS/TMTD & $2,0 / 1,0$ \\
\hline
\end{tabular}

\section{Processo de Mistura}

As misturas NBR/EPDM na composição 70/30 foram feitas em misturador de cilindros Berstoff a $110^{\circ} \mathrm{C}$, com razão de fricção 1:1,1. Primeiramente, a NBR foi processada por $3 \mathrm{~min}$, sendo então adicionado o EPDM e, após $2 \mathrm{~min}, 5,0 \mathrm{phr}$ de agente compatibilizante. Em seguida, a cada 2 minutos, foram adicionados o óxido de zinco, o ácido esteárico, o enxofre e finalmente o MBTS junto com o TMTD. A formulação empregada encontra-se na Tabela 1.

\section{Caracterização das misturas não vulcanizadas}

Durante o processo de mistura foram retiradas alíquotas que continham apenas NBR, EPDM e o agente compatibilizante utilizado, ou seja, antes da adição do sistema de vulcanização. Estas alíquotas foram prensadas por 15 min a $160^{\circ} \mathrm{C}$ e então submetidas à extração com tolueno à quente por 24 horas. Também foram realizados ensaios de extração seletiva com metil etil cetona (MEK) à temperatura ambiente por 5 dias. Ao final da extração as amostras foram secas a vácuo.

\section{Determinação dos Parâmetros Reométricos}

As misturas cruas foram analisadas em reômetro de disco oscilatório a $160^{\circ} \mathrm{C}$ com oscilação de arco $1^{\circ}$, segundo a norma ASTM D-2084. A partir dos reogramas, foram determinados os valores de torque máximo $\left(\mathrm{M}_{\mathrm{H}}\right)$, torque mínimo $\left(\mathrm{M}_{\mathrm{L}}\right)$, tempo de précura $\left(t_{s} 1\right)$ e tempo ótimo de cura $\left(t_{90}\right)$. As misturas foram vulcanizadas em prensa hidráulica aquecida a $160^{\circ} \mathrm{C}$ no tempo ótimo de cura.

\section{Ensaios de inchamento}

Corpos de prova retangulares de $20 \times 10 \times 2 \mathrm{~mm}$, previamente pesados, foram imersos em tolueno e em MEK por sete dias. Ao término do ensaio, os corpos de prova foram pesados e depois secos a vácuo e novamente pesados. $\mathrm{O}$ volume de borracha presente na rede inchada $\left(V_{r}\right)$ foi calculado segundo a equação 1:

$$
V_{r}=\frac{M_{f} \times \rho_{r}^{-1}}{M_{f} \times \rho_{r}^{-1}+\left(M_{i}-M_{f}\right) \times \rho_{s}^{-1}}
$$

onde: $M_{f}$ é a massa seca após o inchamento, $\rho_{r}$ é a densidade da mistura, $M_{i}$ é a massa inchada e $\rho_{s}$ é a densidade do solvente.

\section{Propriedades Mecânicas}

Os ensaios de dureza foram feitos em durômetro do tipo shore A2, segundo a norma ASTM D-224086. Os ensaios de resistência à tração seguiram a norma DIN 53504 empregando corpos de prova do tipo S2, obtidos a partir das placas vulcanizadas com espessura de $2 \mathrm{~mm}$. Os ensaios foram realizados com velocidade de separação das garras de $200 \mathrm{~mm} / \mathrm{min}$. Os ensaios de envelhecimento foram realizados em estufa com circulação forçada de ar a $70{ }^{\circ} \mathrm{C}$ por 72 horas, segundo a norma ASTM D-573-81.

\section{Análises Termo-Dinâmico-Mecânicas}

As análises termo-dinâmico-mecânicas foram realizadas em DMTA (Rheometric Scientific MKIII) no modo flexão, na freqüência de $1 \mathrm{~Hz}$. A faixa de temperatura estudada foi de $-60{ }^{\circ} \mathrm{C}$ até $20^{\circ} \mathrm{C}$ com velocidade de aquecimento de $2^{\circ} \mathrm{C} / \mathrm{min}$. 


\section{Microscopia eletrônica de Varredura}

As amostras foram fraturadas criogenicamente, tingidas com $\mathrm{OsO}_{4}$ durante 3 min e então recobertas com carbono. As análises foram realizadas em microscópio eletrônico de varredura (Zeiss 9600) utilizando detetor de elétrons retroespalhados.

\section{Resultados e Discussão}

Teor de gel

O teor de gel das misturas não-vulcanizadas foi calculado segundo a equação 2 e os resultados encontram-se na Tabela 2.

$$
\text { Teor de gel }(\%)=100 \times\left(\mathrm{M}_{\text {insolúvel }}\right) /\left(\mathrm{M}_{\text {inicial }}\right)
$$

Onde $\mathrm{M}_{\text {inicial }}$ e $\mathrm{M}_{\text {insolúvel }}$ são as massas das amostras antes e após o tratamento com tolueno a quente, respectivamente. Este teste em particular fornece uma noção a respeito da ocorrência de compatibilização reativa. Os grupos mercaptana existentes nas amostras de EVALSH ou EVASH são capazes de reagir com as ligações duplas da borracha nitrílica e/ou EPDM, durante o processo de mistura. A Figura 3 ilustra essa reação com a fase NBR. A ocorrência dessa reação resultará na formação de materiais insolúveis, compostos da porção EVASH e da NBR que reagiu. Como observado na Tabela 2, a mistura não compatibilizada não contém material insolúvel, o que comprova a inexistência de qualquer tipo de interação química entre os elastômeros. Por outro lado, foi detectada a presença de material insolúvel em todas as misturas contendo $5 \mathrm{phr}$ do copolímero funcionalizado com grupos mercaptana, confirmando a reação entre a fase elastomérica e o copolímero funcionalizado. Embora todas as amostras dos agentes interfaciais possuam aproximadamente a mesma proporção de grupos mercaptana, a mistura compa-

Tabela 2. Teor de gel das misturas NBR/EPDM (70:30\% em peso) não vulcanizadas

\begin{tabular}{cc}
\hline & Teor de gel (\%) \\
\hline Não compatibilizada & 0 \\
5 phr de EVALSH & 12 \\
5 phr de EVASH18 & 7,8 \\
5 phr de EVASH28 & 5,3 \\
5 phr de EVASH33 & 4,2 \\
\hline
\end{tabular}

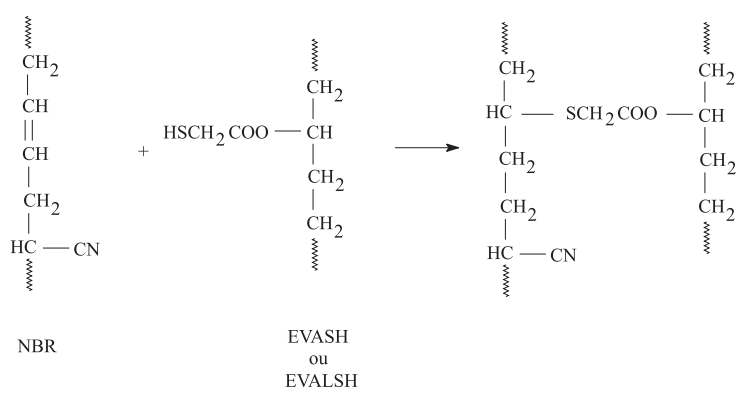

Figura 3. Esquema da reação proposta entre a NBR e o EVA modificado com grupos mercaptana ${ }^{[18]}$.

tibilizada com EVALSH apresentou o maior teor de gel quando comparada às demais. Este resultado pode ser atribuído a uma maior afinidade entre a fase NBR e o EVALSH, devido à possibilidade de formação de ligação de hidrogênio entre os grupos hidroxila livres na amostra de EVALSH e os grupos nitrila na fase NBR. Esta interação proporciona um contato mais íntimo entre os componentes, o que facilita a reação entre os grupos mercaptana e as ligações duplas.

\section{Morfologia das misturas não-vulcanizadas}

Os ensaios de extração seletiva com MEK, da fase NBR, combinados com a microscopia eletrônica de varredura fornecem uma boa noção a respeito da morfologia das misturas. Enquanto a microscopia se limita apenas à superfície de fratura da amostra, o ensaio de extração seletiva é representativo quanto ao todo da amostra. Neste ensaio, a fase NBR é seletivamente extraída. A morfologia é dita cocontínua quando a extração da fase NBR é total e a amostra ainda mantém a estabilidade dimensional. A continuidade da fase NBR foi calculada segundo a equação 3 e os resultados encontram-se na Tabela 3.

$$
(\% \text { Continuidade })_{\mathrm{NBR}}=\left(\mathrm{M}_{\text {solúvel }} / \mathrm{M}_{\text {inicial }}\right)_{\mathrm{NBR}}
$$

Onde $\mathrm{M}_{\text {solúvel }}$ é a massa extraída após tratamento com MEK.

De acordo com os resultados obtidos as misturas NBR/EPDM na razão 70:30\% em peso encontramse cocontínuas, ou seja, tanto a fase EPDM como a fase NBR encontram-se contínuas. Além disso, parte da fase NBR já começa a se dispersar ao longo da fase EPDM. A adição dos agentes interfaciais promove uma maior dispersão da fase NBR.

As misturas não-vulcanizadas foram criogenicamente fraturadas e em seguida tingidas com solução de $\mathrm{OsO}_{4}$. Considerando que o contato da amostra com a solução de $\mathrm{OsO}_{4}$ foi rápido, a fase NBR, que possui maior teor de ligações duplas é tingida 
Tabela 3. Continuidade das fases NBR e EPDM em função do agente compatibilizante empregado.

\begin{tabular}{ccc}
\hline & \multicolumn{2}{c}{ Continuidade, \% } \\
\cline { 2 - 3 } & Fase NBR & Fase EPDM \\
\hline n-comp. & 97 & 100 \\
EVALSH18 (5 phr) & 92 & 100 \\
EVASH18 (5 phr) & 90 & 100 \\
EVASH28 (5 phr) & 90 & 100 \\
EVASH33 (5 phr) & 88 & 100 \\
\hline
\end{tabular}

preferencialmente. As micrografias são apresentadas na Figura 4. A região clara se refere à fase tingida de NBR e a região escura se refere à fase EPDM. As micrografias confirmam os resultados da extração seletiva. Ainda é possível observar que o tamanho dos domínios da fase NBR dispersa são menores para a mistura compatibilizada com o EVA18SH.

\section{Parâmetros Reométricos}

Os dados reométricos são apresentados na Tabela 4. A adição de EVA transesterificado reduz discretamente os valores de torque máximo e mínimo. Quanto maior o teor de acetato de vinila menor é o valor de torque máximo e mínimo, sugerindo uma melhor processabilidade da mistura. Por outro lado, a adição de EVALSH não promoveu alteração alguma nos valores de torque quando comparada à mistura não-compatibilizada. A resistência à pré-cura, denotada pelo valor de $t_{s} 1$, é menor para as misturas compatibilizadas em decorrência da presença de grupos mercaptan, os quais atuam como aceleradores de vulcanização. O tempo ótimo de cura também é menor para as misturas compatibilizadas.

Tabela 4: Parâmetros reométricos e dureza da misturas NBR/EPDM (70:30\% em peso)

\begin{tabular}{cccccc}
\hline & n-comp & EVALSH & EVASH & EVASH & EVASH \\
& & & 28 & 33 \\
\hline $\mathrm{M}_{\mathrm{L}}^{\mathrm{a}}$ & 3,6 & 3,7 & 3,5 & 3,3 & 3,0 \\
$\mathrm{M}_{\mathrm{H}}^{\mathrm{b}}$ & 24,4 & 24,5 & 22,7 & 22,0 & 21,5 \\
$\mathrm{M}_{\mathrm{H}}-\mathrm{M}_{\mathrm{L}}$ & 20,8 & 20,8 & 19,2 & 18,7 & 18,5 \\
$\mathrm{t}_{\mathrm{s}} 1(\min )$ & 3,5 & 2,8 & 2,5 & 2,5 & 2,4 \\
$\mathrm{t}_{90}(\min )$ & 6,1 & 5,6 & 5,4 & 4,4 & 4,5 \\
Dureza & 48 & 52 & 51 & 51 & 51 \\
\hline
\end{tabular}

a Torque mínimo em lbf.in, ${ }^{\mathrm{b}}$ Torque máximo em lbf.in (a)

(b)

(c)

Figura 4. Micrografias das misturas NBR/EPDM (70:30\% em peso) não vulcanizadas.

Os valores da diferença $\mathrm{M}_{\mathrm{H}}-\mathrm{M}_{\mathrm{L}}$, assim como a dureza, fornecem uma idéia a respeito da extensão da formação de ligações cruzadas durante o processo de vulcanização. Embora tenham sido constatadas pequenas variações no valor de $\mathrm{M}_{\mathrm{H}}-\mathrm{M}_{\mathrm{L}}$ para as misturas compatibilizadas com EVA transesterificado, 
os valores de dureza mantiveram-se constantes e praticamente iguais à dureza da mistura compatibilizada com EVALSH. Portanto, é mais adequado relacionar os valores de $\mathrm{M}_{\mathrm{H}}-\mathrm{M}_{\mathrm{L}}$ com a natureza dos agentes compatibilizantes. Esta redução dos valores de $\mathrm{M}_{\mathrm{H}}-\mathrm{M}_{\mathrm{L}}$ pode ser atribuída às diferentes características de flexibilidade dos agentes compatibilizantes. O EVALSH é mais rígido do que os demais, em função da presença dos grupos hidroxila no primeiro passo da rota de funcionalização, enquanto que os demais agentes compatibilizantes, oriundos da transesterificação, têm seus grupos acetato preservados e são conseqüentemente mais flexíveis, facilitando o processamento.

\section{Ensaios de inchamento}

Os ensaios de inchamento foram realizados em tolueno e metil-etil-cetona (MEK), sendo os resultados apresentados na Figura 5. Pode-se observar que, para os ensaios realizados em tolueno, as misturas compatibilizadas apresentam valores de $\mathrm{Vr}$ mais altos do que a mistura não-compatibilizada. As misturas compatibilizadas com EVA transesterificado apresentam valores de Vr ligeiramente maiores do que a mistura compatibilizada com EVALSH, principalmente quando EVASH 28 ou 33 são usados. Estes resultados sugerem que, de um modo geral, as misturas compatibilizadas encontram-se melhor reticuladas do que a mistura não-compatibilizada. $\mathrm{O}$ EPDM melhor reticulado pode estar agindo de maneira semelhante a uma carga, restringindo o inchamento da fase NBR. Pode-se imaginar que os agentes compatibilizantes agem não só de forma a melhorar a interação entre a NBR e o EPDM, como também distribuem melhor a reticulação na fase EPDM. Por outro lado, quando o ensaio de inchamento foi feito em MEK, no qual incha apenas a fase NBR, todas as misturas apresentaram valores de $\mathrm{Vr}$ bem próximos e praticamente constantes, mostrando que a reticulação da fase NBR não é afetada pela presença dos agentes compatibilizantes.

Todas as misturas apresentaram perda de massa ao final dos ensaios de inchamento tanto em tolueno como em MEK. Tais perdas ficaram em torno de $10-12 \%$ e $2-3 \%$, respectivamente. Isto significa que cerca de 8-9 \% da massa perdida é EPDM, em decorrência da grande incompatibilidade entre velocidades de cura da NBR e do EPDM. Neste ponto, parece que os agentes compatibilizantes atuam de

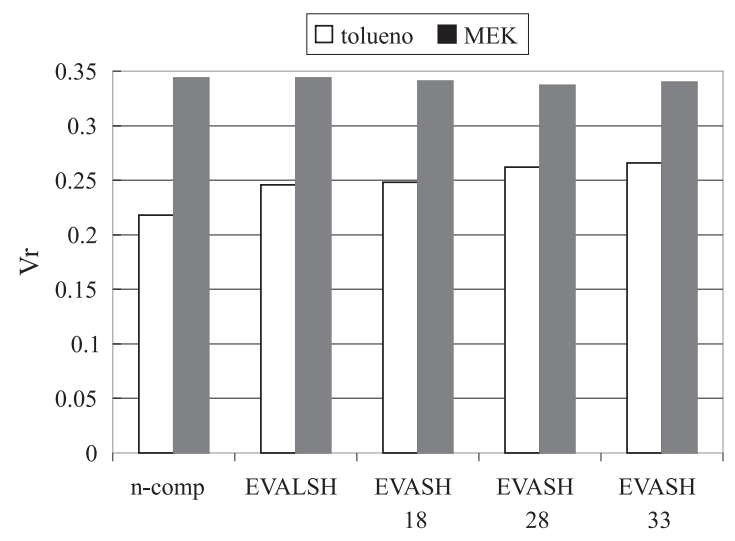

Figura 5. Volume de borracha presente na rede inchada (Vr) para os ensaios de inchamento realizados em tolueno e MEK

forma a distribuir melhor a reticulação na fase EPDM do que propriamente reticular mais tal fase.

\section{Análise Termo-dinâmico-mecânica}

As curvas obtidas nas análises termo-dinâmicomecânicas apresentaram dois picos de máximo na curva do fator de amortecimento $(\tan \delta)$ : um referente à transição vítrea do $\mathrm{EPDM}$ a $-44,6{ }^{\circ} \mathrm{C}$ e outro referente à transição vítrea da NBR a $-8,9^{\circ} \mathrm{C}$, confirmando a incompatibilidade entre o EPDM e a NBR.

A Figura 6 ilustra a variação do fator de amortecimento com a temperatura para a fase NBR. $\mathrm{A}$ adição de EVALSH à mistura promove algumas mudanças na curva de $\tan \delta$. Nota-se um ligeiro alargamento e amortecimento do pico referente à transição vítrea da NBR. Além disso, constata-se um pequeno deslocamento da $\mathrm{Tg}$ para $-13,1^{\circ} \mathrm{C}$, por conta do alargamento do pico. A adição de EVASH 18 não produz alterações significativas na curva de $\tan \delta$, sendo apenas observado um ligeiro deslocamento da Tg para $-10,7^{\circ} \mathrm{C}$. As curvas de tan $\delta$ para as misturas compatibilizadas com EVASH 28 e 33 são praticamente idênticas à curva da mistura não-compatibilizada. A única diferença observada é um pequeno deslocamento da Tg para $-11,1^{\circ} \mathrm{C}$, quando a mistura é compatibilizada com EVASH 33.

A Figura 7 ilustra o comportamento da curva do fator de amortecimento $(\tan \delta)$ da fase EPDM para as misturas estudadas. A mistura compatibilizada com EVALSH apresenta um leve deslocamento da região de transição vítrea do EPDM para uma temperatura mais baixa. Além disso, observa-se um amortecimento do pico referente a esta transição. É interessante notar que esta mistura é a única em que 
Tabela 5. Propriedades termodinâmico-mecânicas da mistura NBR/ EPDM (70:30\% em peso)

\begin{tabular}{ccccc}
\hline & \multicolumn{2}{c}{ Fase EPDM } & \multicolumn{2}{c}{ Fase NBR } \\
\cline { 2 - 5 } & $\mathrm{Tg},{ }^{\circ} \mathrm{C}$ & $\operatorname{Tan} \delta$ & $\mathrm{Tg},{ }^{\circ} \mathrm{C}$ & $\tan \delta$ \\
\hline n-comp. & $-44,6$ & 0,200 & $-8,9$ & 0,931 \\
EVALSH & $-46,4$ & 0,168 & $-13,1$ & 0,905 \\
EVASH 18 ${ }^{\mathrm{a}}$ & $-45,6$ & 0,158 & $-10,7$ & 0,930 \\
EVASH 28 & $-42,9$ & 0,157 & $-8,6$ & 1,020 \\
EVASH 33 & $-48,5$ & 0,138 & $-11,1$ & 0,971 \\
\hline a
\end{tabular}

${ }^{a} 5,0 \mathrm{phr}$ de agente compatibilizante

já é possível observar o início da transição vítrea referente à fase NBR. Comportamento bem semelhante é exibido pela mistura compatibilizada com EVASH 18, sendo que o amortecimento é ligeiramente maior. Este amortecimento da curva de $\tan \delta$ na região de transição vítrea do EPDM sugere que esta fase esteja melhor reticulada nas misturas compatibilizadas, o que leva a uma redução nos valores de máximo da $\tan \delta$. Os resultados de inchamento em tolueno justificam este comportamento. É interessante notar que a mistura compatibilizada com EVASH 28 apresenta não só uma redução no valor máximo da curva de $\tan \delta$ mas também um discreto deslocamento da $\mathrm{Tg}$ para $42,6{ }^{\circ} \mathrm{C}$. Por outro lado, a mistura compatibilizada com EVASH 33 apresenta um deslocamento da $\mathrm{Tg}$ para $-48,5^{\circ} \mathrm{C}$, embora também o valor máximo da curva de $\tan \delta$ tenha sido reduzido. O perfil das curvas de $\tan \delta$ na região de transição vítrea da fase EPDM sugere que os agentes compatibilizantes alteram de forma mais acentuada o comportamento da fase EPDM, exceto o EVALSH que também modifica o comportamento da fase NBR.

Figura 6. Curva do fator de amortecimento $(\tan \delta)$ da fase NBR para as misturas NBR/EPDM (70:30\% em peso)
Figura 7. Curva do fator de amortecimento $(\tan \delta)$ da fase EPDM para as misturas NBR/EPDM (70:30\% em peso).

A Tabela 5 apresenta um resumo sobre as principais propriedades termodinâmico-mecânicas.

\section{Morfologia das Misturas Vulcanizadas}

As misturas vulcanizadas foram criogenicamente fraturadas e em seguida tingidas com solução de $\mathrm{OsO}_{4}$. As micrografias são apresentadas na Figura 8. As partes claras se referem à fase NBR e as partes escuras se referem à fase EPDM.

A mistura não compatibilizada apresenta uma morfologia onde é possível distinguir grandes domínios segregados de cada uma das fases, não se podendo distinguir a matriz da fase dispersa, resultando numa morfologia do tipo cocontínua, embora a fração de EPDM na mistura seja de apenas $30 \%$. A adição de EVALSH parece promover uma quebra dos domínios da fase NBR, melhorando a distribuição desta. Neste caso, ainda é possível observar uma morfologia do tipo cocontínua, mas já começa a observar-se parte da fase NBR dispersa ao longo da fase EPDM, a qual passa a ser matriz. No entanto os domínios de NBR ainda são grandes e apresentam uma grande heterogeneidade no tamanho e na forma.

A adição de EVA transesterificado promove uma distribuição mais homogênea da fase EPDM ao longo da fase NBR, embora os domínios ainda sejam alongados. O tamanho destes é menor quando comparado ao das demais misturas, demonstrando uma uniformidade maior. Não se verifica a segregação que ocorre na mistura não compatibilizada. Este comportamento se acentua à medida que aumenta o teor de acetato de vinila no agente compatibilizante. Desta forma, pode-se propor que a maior flexibilidade proveniente do maior teor de acetato de vinila e também da ausência de grupos hidroxila na cadeia 
(a)

(d) (b)

(c)

(d)

Figura 8. Microscopia eletrônica de varredura das misturas NBR/EPDM (70:30\% em peso) compatibilizadas e não-compatibilizada.

do EVA, permitem que este atue facilitando o processamento e favorecendo a distribuição mais homogênea da fase EPDM.

\section{Propriedades Mecânicas}

A Tabela 6 apresenta os resultados de resistência à tração. A presença dos agentes compatibilizantes resulta no aumento do alongamento na ruptura, sendo os maiores valores encontrados para as misturas compatibilizadas com EVASH 18 e 28. Entretanto os valores de tensão na ruptura são bastante próximos para as misturas não-compatibilizada e compatibilizadas com EVA transesterificado, embora estas últimas apresentem valores de tensão na ruptura ligeiramente mais altos. É interessante notar que a mistura compatibilizada com EVALSH apresenta tensão na ruptura inferior às demais misturas. Neste caso, a característica mais rígida do EVALSH, quando comparado ao EVA transesterificado, pode ter produzido uma interface pouco flexível. Os valores de tensão na ruptura estão de acordo com a morfologia encontrada para essas misturas.
A resistência ao envelhecimento das misturas NBR/EPDM foi avaliada em termos de porcentagem de retenção da tensão e do alongamento na ruptura. Estes resultados encontram-se na Tabela 6. Vale ressaltar que a mistura NBR/EPDM não compatibilizada é bastante resistente ao envelhecimento, sendo capaz de praticamente manter as suas propriedades iniciais. Este comportamento é compatível com o proposto na literatura, segundo a qual o EPDM confere maior resistência ao envelhecimento aos elastômeros diênicos. As misturas

Tabela 6: Resistência à tração e ao envelhecimento das misturas NBR/ EPDM (70/30)

\begin{tabular}{cccccc}
\hline & n-comp & EVALSH & $\begin{array}{c}\text { EVASH } \\
\mathbf{1 8}\end{array}$ & $\begin{array}{c}\text { EVASH } \\
\mathbf{2 8}\end{array}$ & $\begin{array}{c}\text { EVASH } \\
\mathbf{3 3}\end{array}$ \\
\hline$\sigma, \mathrm{MPa}$ & 2,96 & 2,56 & 3,44 & 3,35 & 3,35 \\
$\Delta \sigma, \%$ & 98 & 108 & 96 & 100 & 108 \\
$\varepsilon, \%$ & 300 & 325 & 400 & 400 & 350 \\
$\Delta \varepsilon, \%$ & 106 & 92 & 90 & 91 & 94 \\
\hline
\end{tabular}

$\Delta \sigma=\%$ de retenção do valor inicial de $\sigma$ $\Delta \varepsilon=\%$ de retenção do valor inicial de $\varepsilon$ 
compatibilizadas são capazes de reter e até mesmo melhorar os valores de tensão na ruptura, este último caso sendo observado para as misturas compatibilizadas com EVALSH e EVASH 33. Por outro lado, os valores de alongamento na ruptura são ligeiramente reduzidos, sugerindo um processo de enrijecimento destas misturas ou ainda um fenômeno de pós-cura, considerando-se que esta redução é pequena e comparável ao erro do ensaio.

\section{Conclusões}

Os grupos mercaptana se mostraram efetivos na reação com a porção butadieno da fase NBR, como foi constatado através da formação de material insolúvel nos ensaios para determinação do teor de gel. Estes resultados sugeriram a viabilidade da compatibilização reativa.

A mistura NBR/EPDM na composição 70/30 encontra-se cocontínua, isto é, ambas as fases são contínuas. Esta morfologia da mistura não-vulcanizada foi modificada pela presença dos agentes interfaciais, que agiram de forma a reduzir a quantidade de fase NBR originalmente presente na forma contínua. Além disso, a presença de EVASH 18 reduziu o tamanho dos domínios da fase NBR dispersa. Acredita-se que a interface desta mistura se encontre mais estabilizada contra a coalescência.

Lembrando que há formação in situ do graft NBREVALSH ou NBR-EVASH 18, estes precisam migrar até a interface para que aconteça a compatibilização e, portanto, a viscosidade destes copolímeros é um fator importante. Tanto o EVALSH como o EVASH 18 são copolímeros semicristalinos. Entretanto, o primeiro se apresenta mais cristalino devido à presença dos grupos $\mathrm{OH}$, os quais através de interações do tipo ligação de hidrogênio favorecem a cristalização. Desta forma, acredita-se que a maior cristalinidade do EVALSH prejudique a migração do copolímero NBR-g-EVALSH até a interface, devido à sua menor mobilidade.

A adição de EVA modificado com grupos mercaptana reduziu o tempo ótimo de cura e pré-cura da mistura NBR/EPDM. Os grupos mercaptana fazem parte de uma conhecida classe de aceleradores. Além disso, o EVA modificado via transesterificação (rota 2) atuou também como agente de processamento, devido à sua maior flexibilidade, diminuindo os valores de torque máximo e mínimo nos ensaios realizados em reômetro de disco oscilatório. A formação de ligações cruzadas também foi afetada pela presença dos agentes compatibilizantes, conforme pôde ser verificado pelos maiores valores de Vr obtidos nos ensaios de inchamento em tolueno. Ainda foi possível notar, através dos ensaios de inchamento em MEK, que a reticulação da fase NBR não foi significativamente afetada pela presença dos agentes compatibilizantes. Estes, porém, melhoram a reticulação da fase EPDM, principalmente quando o EVASH 28 transesterificado foi utilizado.

As análises dinâmico-mecânicas das misturas revelaram que os agentes compatibilizantes atuam na fase EPDM, reduzindo a mobilidade desta. O EVASH 33, entretanto, desloca a Tg desta fase para temperaturas mais baixas, sugerindo que, a partir deste teor de acetato de vinila, ocorra um fenômeno de plastificação da fase EPDM. Contudo, a fase NBR só sofreu alterações significativas quando o EVALSH foi empregado. É importante ressaltar a concordância destes resultados com os resultados dos ensaios de inchamento.

A morfologia das misturas vulcanizadas compatibilizadas com EVA transesterificado se revelou mais refinada, com uma distribuição mais homogênea da fase EPDM ao longo da fase NBR. Este efeito foi mais pronunciado para o EVA contendo maiores teores de acetato de vinila. Enquanto nestas misturas havia uma distribuição mais homogênea das fases, na mistura compatibilizada com EVALSH observouse uma certa heterogeneidade no tamanho e nas formas dos domínios da fase NBR, a qual já começava a se apresentar parcialmente dispersa. A comparação das morfologias das misturas não-vulcanizadas e vulcanizadas sugere que a reticulação reverte o processo de inversão de fase nas misturas compatibilizadas com o EVA transesterificado.

As misturas compatibilizadas com EVA modificado via transesterificação tiveram maior tensão e alongamento na ruptura, enquanto que o EVALSH apresentou propriedades inferiores às da mistura nãocompatibilizada, em função de uma interface mais rígida. A resistência ao envelhecimento da mistura não-compatibilizada foi excelente, confirmando a capacidade do EPDM de melhorá-la. Além disso, as misturas compatibilizadas foram capazes de manter e até mesmo melhorar a resistência à tração após o envelhecimento, até mesmo pela presença de grupos mercaptana que capturam os radicais livres produ- 
zidos durante o envelhecimento, evitando o prosseguimento da reação de quebra de cadeia.

\section{Agradecimentos}

Os autores agradecem a CAPES, CEPG-UFRJ, CNPq e PADCT/CNPq (Proc. no620132/98-1) pelo apoio financeiro recebido e a NITRIFLEX S.A. e a DSM pela doação dos elastômeros.

\section{Referências Bibliográficas}

1. von Hellens, W. -Kauts. Gummi Kunsts., 47, 124, (1994).

2. von Duin, M.; Krans, J. C. J.; Smedinga, J. - Kauts. Gummi Kunsts., 46, 445 (1993).

3. Coran, A.Y. - Rubber Chem. Technol., 61, 281, (1988).

4. Struckmeyer, F.; Hofmann, W. - Intern. Polym. Sci. Technol., 16, 1, (1989).

5. Baranwal, K. C.; Son, P. N. - Rubb. Chem. Technol., 47, 88, (1974).

6. Radusch, H. J.; Lämmer, E.; Spirk, E.; Dudik, M. - Kauts. Gummi Kunsts., 46, 703 (1993).

7. Lohmar, J. - Kauts. Gummi Kunsts., 39, 1065, (1986).

8. Setua, D. K.; Pandey, K. N.; Saxena, A. K.; Mathur, G. N. - J. Appl. Polym. Sci., 74, 480, (1999).

9. Setua, D. K.; White, J. L. - Polym. Eng. Sci., 31, 1742, (1991).
10. Setua, D. K.; White, J. L. - Kauts. Gummi Kunsts., 44, 137, (1991).

11. Setua, D. K.; White, J. L. - Kauts. Gummi Kunsts., 44, 542, (1991).

12. Setua, D. K.; White, J. L. - Kauts. Gummi Kunsts., 44, 821, (1991).

13. Jansen, P.; Amorim, M.; Gomes, A. S.; Soares, B. G. J. Appl. Polym. Sci., 58, 101, (1995).

14. Jansen, P.; Gomes, A. S.; Soares, B. G. - J. Appl. Polym. Sci., 61, 591, (1996).

15. Tavares, M. I. B.; Jansen, P.; Soares, B. G. - Polym. Bull., 37, 215, (1996).

16. Jansen, P.; Soares, B. G. - Polym. Degrad. Stab., 52, 95, (1996).

17. Jansen, P.; Silva, E. F.; Gomes; A. S., Soares, B. G. Macromol. Rep., 32, 671, (1995).

18. Jansen, P.; Soares, B. G. - J. Appl. Polym. Sci., 79, 193, (2001).

19. Soares, B. G.; Alves, F. F.; Oliveira, M. G.; Moreira, A.C.F.; Garcia, F. G.; Lopes, M. F. S. - Europ. Polym. Journal, 37, 1577, (2001).

20. Dutra, R. C. L.; Lourenço, V. L.; Diniz, M. F.; Azevedo, M. F. P.; Barbosa, R. V.; Soares, B. G. - Polym. Bull., 36, (1996).

Recebido: $10 / 08 / 01$

Aprovado: 20/06/02 